\title{
A Survey on Rate Adaptation Algorithms for Effective Resource Utilization in LTE Advanced
}

\author{
Diksha Duggal and Jyoteesh Malhotra \\ ECE Department, Guru Nanak Dev University, RC Jalandhar, Punjab, India \\ dikshaduggal95@gmail.com,jyoteesh@gmail.com
}

\begin{abstract}
LTE Advanced has brought revolution in the field of wireless communication technology by providing high data rates of up to 1Gbps. There is a need of increased data rates these days that may support the delivery of richer mobile multimedia contents to the end users. Rate adaptation is one of the key enabling technology in the success of LTE Advanced that has cited attraction of academia and research communities in recent years. Rate adaptation is the determination of the optimal data transmission rate most suitable for current prevailing wireless channel conditions. To maximize the performance of wireless devices, it is important to select the best appropriate rate hence rate adaptation algorithms (RAA's) are required. This work includes a comprehensive survey on various types of RAA's in LTE-A for effective resource utilization. Depending on the channel conditions various performance parameters such as throughput, power consumption and latency have been studied. It has been observed from the literature survey that many proposals have been published on LTE-A. Sill there is a need of research to be carried in LTE-A as this technology is not fully matured so open issues and future scope are also raised in this work.
\end{abstract}

Keywords: LTE Advanced, Rate Adaptation Algorithms, Resource utilization

\section{Introduction}

LTE advanced has more advanced set of standards than LTE that promises to deliver true $4 \mathrm{G}$ speeds. LTE supports higher peak rates, throughput, coverage and lower latencies. It offers peak download speed of $1 \mathrm{Gbps}$. Rate adaptation is the determination of the optimal data transmission rate that is suitable for current prevailing wireless channel conditions. It includes assessing channel conditions and accordingly adjusting the rate. Rate adaptation in LTE Advance is fairly challenging due to wild channel conditions fluctuations. This performance may be defined to be the raw throughput, power saving, low loss or via other performance parameters.

In present scenario it is crucial to select correct rate that may maximize the performance of wireless devices hence RAA's are required. Considering the importance of RAA lot many technical papers have been published by researchers. In this work also a survey of rate adaptation algorithms for effective resource utilization in LTE-advanced has been done. The paper is organized as technical background in section(2), literature survey in section(3), tabular comparison of various RAA's in section(4), open issues and future scope in $\operatorname{section}(5)$.

\section{Technical Background}

Algorithms that adapt the parameters of transmission to the channel conditions may be designed to optimize several parameters depending on the network topology and the type of devices used: 
$\diamond$ Power consumption- Mobile devices that implement LTE advance technology usually have a fixed energy budget due to finite battery life. Hence it is of utter importance to minimize the amount of energy consumed.

$\diamond$ Throughput- Higher transmission rates often provide potential throughput but usually have higher Bit Error Rates (BERs). Higher BER's require more retransmissions for error-free transmissions which thereby results in decreasing the application level throughput.

$\diamond$ Latency- Low latency systems allow the implementation of per packet adaptation algorithms. High latency system requires periodic analysis of the transmission characteristics and updates to the transmission parameters.

\section{Literature Survey}

LTE-Advanced supports higher peak rates, higher throughput, coverage, and lower latencies thereby resulting in a better user experience. There is a greater need of increased data rates these days that may support the delivery of richer mobile multimedia contents to the end users. So the various rate adaptations algorithms (RAA) to maximize the performance of LTE Advance are presented in this work.

\subsection{Adaptive Auto Rate Fallback (AARF) Algorithm}

AARF is an algorithm with the goal of performing better in stable channel conditions. AARF increases several consecutive successful transmissions it needs before it tries to send a transmission at a higher rate than the present prevailing one. It does this by remembering the several failed attempts to probe the channel at a higher rate and each time the probe transmission fails [1], the algorithm multiplies the several consecutive successful transmissions by 2 to a count of maximum of up to 50. If during the current transmission packet fails twice, it decreases the transmission rate by one step and resets the consecutive successful bit transmission counter to a value of 10. AARF have characteristics as shown-

- Reducing the rate

- AARF reduces the current rate if consecutive 2 packets are lost.

- They fall back to the next lower rate if probing packet fails.

- Frequency of best rate changes.

- Current rate can change upwards as quickly as every 10 packets.

- Current rate can change downwards as quickly as every 2 packets.

To better reflect the channel conditions, the threshold is continuously changed at run-time in AARF algorithm and hence it performs better in stable channel conditions.

\subsection{Adaptive Multi-Rate Retry (AMRR) Algorithm}

AMRR is an algorithm based on ARF with an additional Binary Exponential Back off (BEB) much as AARF. The Atheros AR 5212 chip-set exports a mechanism to drivers called Multi-Rate Retry (MRR).The MRR is a descriptor table that is being sent to the hardware along with the data to be transmitted. The descriptor table is populated with 4 rates used when transmitting the frame. Each rate is accompanied with a count value stating the times each rate is attempted to send. To get the BEB in AMRR, the values of the count fields in the rate/transmission count are set to one, $\mathrm{c} 0=1, \mathrm{c} 1=1, \mathrm{c} 2=1$ and $\mathrm{c} 3=1$ [1]. The transmission rates are chosen based on the current transmission rate and the minimum rate of the wireless medium used. R3 is always set to the minimum value of transmission rate and R1 and R2 are set to two rates just below R0. In this algorithm the 
probability of trying a rate set that requires numerous retransmissions is greatly diminished.

\subsection{Robust Rate Adaptation Algorithm (RRAA+)}

RRAA+ algorithm offers a unique feature of convergence. This algorithm works with high and low thresholds of loss rate based on a current data rate selected. RRAA+ maintains for each bit rate the probability that the transmitter transitions to this bit rate from a lower bit rate. Every time the loss at a bit rate exceeds the high threshold, the probability of returning to this bit rate is reduced by a factor A1 before transitioning to the next lower rate. In each interval if the bit rate causes the packet loss to be below the low threshold, the probability of this bit rate is increased by a factor A2. The multiplicative increase multiplicative decrease (MIMD) parameters A1 and A2 are used to keep the algorithm stable. Adaptive request to send (ARTS) mechanism is another unique feature of RRAA+. To prevent receiver side interference the RTS is estimated. The adaptive RTS mechanism detects any packet losses that are happening due to collisions as opposed to degraded channel conditions. RRAA+ outperforms with very high throughput improvement.

\subsection{Optimal Power and Rate Adaptation (OPRA) Algorithm}

In OPRA scheme, the transmission power and rate is matched to the varying channel condition through use of a multiplexed multiple codebook design. This leads to the highest achievable capacity and to achieve this side information of the channel fade level (i.e., CSI) tracked at the receiver must be conveyed to the transmitter on the feedback path for power and rate adaptation in accordance with the time-varying nature of the channel fading.

\subsection{Optimal Rate Adaptation with Fixed Transmit Power (ORA) Algorithm}

ORA algorithm is used to adapt the rate as per changing channel conditions. The channel capacity when CSI (channel state information) is available only at the receiver [5] is given as-

$$
\bar{C}_{\text {ORA }}=\frac{\beta}{\ln 2} \int_{0}^{\infty} \ln (1+\gamma) f_{\gamma}(\gamma) d \gamma
$$

Since it is generally difficult or expensive to acquire fast, reliable feedback from the receiver to the transmitter, above relation is a useful capacity bound that is applicable to many situations encountered in practical operating conditions. It is interesting to point out that the capacity increases with the increasing diversity order regardless of the fading channel model or diversity combining technique employed.

\subsection{Collision Aware Rate Adaptation-Rate Increase Algorithm:}

The key idea of CARA-RI is to introduce collision aware rate increase decisions into CARA BASIC. It combines adaptive RTS/CTS exchange with Clear Channel Assessment (CARA) functionality to detect frame collisions. CARA-RI only resets $m$ when the frame loss occurs with RTS/CTS preceding the transmission attempt. Hence $\mathrm{m}$ is reset only when the frame loss may be clearly identified as a channel-error caused failure. Thereby CARA-RI adjusts the transmission rate more quickly to the improving wireless channel condition than CARA BASIC. 


\subsection{Collision Aware Rate Adaptation Hidden Detection (CARA-HD) Algorithm}

CARA-HD incorporates a hidden station based detection mechanism. In the case of infrastructure based WLAN system where all stations may hear the Access Point (AP), but stations may be hidden to each other. Let A and B denote a pair of hidden stations in the network. Although A may not hear the data transmission from the access point to B. By looking into either the frame content or the frame length information carried in the physical layer header, A should be able to figure out that this is a Back frame transmission and consequently if $\mathrm{A}$ did not hear any data frame transmission associated with this acknowledgement; it may claim detection of a hidden station. In CARA-HD as the several contending stations increases the performance gap among them becomes more significant.

\subsection{Semi Open Loop Rate Adaptation Algorithm}

This is a method for quickly and accurately adapting a rate in a MIMO system while minimizing system overhead. This system may include first and second nodes in which transmissions from the first node to the second node are on a "downlink channel" and transmissions from the second node to the first node are on an "uplink channel". Each node in the MIMO system may include multiple transmitters and receivers. In this algorithm, the first node may estimate the uplink channel using a packet sent by the second node to the first node. This uplink channel may be transposed so as to analyze an estimated downlink channel. The first node may use transmitter and receiver characteristics from both the first and second nodes and the estimated downlink channel so as to accurately adapt the rate. Notably, the receiver characteristics may include the sensitivity of the second node. In one embodiment, using the transmitter and receiver characteristics may include computing a post- detection SNR of the second node based on the estimated downlink channel. This post-detection SNR may be adjusted with a transmit output power of the second node for a data rate of the packet received. After adjusting, an estimated post-detection SNR for each rate at the second node may be computed by using the transmitter power per rate of the first node and hence building a sensitivity table for the second node. If the transmitter EVM is not negligible, then the estimated postdetection SNR for each rate at the second node may be adjusted with a transmitter EVM per power of the first and second nodes. This algorithm quickly and accurately adapts rate in MIMO system while minimizing system overhead.

\subsection{Coordinated Multipoint Transmission and Reception (COMP) Rate Adaption Algorithm}

COMP rate adaptation algorithm better supports multiplexing transmission and expands the coverage of high data rate services. The primary principle of COMP is to coordinate multiple geographically distributed base stations to serve UE more efficiently. Depending on a UE unit's capability of measuring and returning the CSI between itself and multiple BS's, for high data rate downlink transmission three types of schemes have been proposed [3]. These RA techniques are Coordinated Scheduling or Coordinated beam forcing (CS/CB), Joint Transmission (JT) and Transmission Point Selection (TPS).

\subsection{Carrier Aggregation (CA) Algorithm}

CA rate adaptation algorithm takes advantage of the fact that assigning more spectrum to the user increases the data rate [4]. Here primary cell handles mobility, user data, physical control signaling and broadcast information. Secondary cell provides higher data throughout. Various types of $\mathrm{CA}$ are Intra-Band Contiguous CA, Inter-Band Non Contiguous CA and Intra-Band Non Contiguous CA [7]. Carrier Aggregation is a technique of aggregating multiple carriers of same or varying bandwidths so as to increase the overall transmission bandwidth and thereby increasing the bit rate. The component 
carrier may have a bandwidth of $1.4,3,5,10,15$ or $20 \mathrm{MHz}$ and a maximum of five component carriers may be aggregated, hence the maximum aggregated bandwidth is $100 \mathrm{MHz}$ [11]. The developments of the carrier aggregation in Release 10 (LTE-A) specifications are continuing to follow, providing further-enhanced capacity and higher peak data rates.

\section{Evaluation of RAA's Representing Current State of Art}

\begin{tabular}{|c|c|c|c|}
\hline $\begin{array}{l}\text { Sr. } \\
\text { No. }\end{array}$ & $\begin{array}{c}\text { Rate Adaption } \\
\text { Algorithm }\end{array}$ & Merits & Demerits \\
\hline 1. & $\begin{array}{l}\text { Adaptive } \text { Auto Rate } \\
\text { Fallback } \quad \text { R.A. } \\
\text { Algorithm }\end{array}$ & $\begin{array}{l}\text { - AARF performs better in stable } \\
\text { channel conditions. } \\
\text { To better reflect the channel } \\
\text { conditions, the threshold is } \\
\text { continuously changed at runtime. }\end{array}$ & $\begin{array}{l}\text { - One successful probe } \\
\text { triggers incorrect rate } \\
\text { increase } \\
\text { One unsuccessful } \\
\text { probe incurs higher } \\
\text { penalty on future rate } \\
\text { adaptation }\end{array}$ \\
\hline 2. & $\begin{array}{ll}\text { Adaptive } & \text { Multi } \\
\text { Rate Retry } & \text { R.A. } \\
\text { Algorithm } & \end{array}$ & $\begin{array}{l}\text { - } \text { BEB based adaptive mechanism } \\
\text { results in improving throughput. } \\
\text { - Here probability of trying a rate set } \\
\text { which requires numerous } \\
\text { retransmissions is greatly } \\
\text { diminished. }\end{array}$ & $\begin{array}{l}\text { binary exponential } \\
\text { back-off tends to be } \\
\text { too conservative in } \\
\text { adapting rates }\end{array}$ \\
\hline 3. & $\begin{array}{ll}\text { Robust } & \text { Rate } \\
\text { Adaptation } & \\
\text { Algorithm } & \text { Plus } \\
\text { (RRAA+) } & \end{array}$ & $\begin{array}{l}\text { - RRAA+ outperforms with very } \\
\text { high throughput improvement. } \\
\text { - Quick responsiveness }\end{array}$ & $\begin{array}{l}\text { - More overhead with } \\
\text { RTS filter } \\
\text { - Needs more training } \\
\text { and comparison so as } \\
\text { to get optimized } \\
\text { Parameters }\end{array}$ \\
\hline 4. & $\begin{array}{l}\text { Optimal Power and } \\
\text { Rate Adaptation } \\
\text { Algorithm }\end{array}$ & $\begin{array}{l}\text { - OPRA leads to high achievable } \\
\text { capacity with available channel } \\
\text { state information }\end{array}$ & - $\quad$ Increased overheads \\
\hline 5. & $\begin{array}{lr}\text { Optimal } & \text { Rate } \\
\text { Adaptation } & \text { with } \\
\text { fixed } & \text { transmit } \\
\text { power } & \end{array}$ & $\begin{array}{l}\text { - ORA algorithm is used to adapt the } \\
\text { rate according to changing channel } \\
\text { conditions. }\end{array}$ & - $\quad$ Variations in SNR \\
\hline 6. & $\begin{array}{l}\text { CARA Rate } \\
\text { Increase Algorithm }\end{array}$ & $\begin{array}{l}\text { - CARA-RI increases transmission } \\
\text { rate more proactively hence yields } \\
\text { higher aggregate throughput. }\end{array}$ & $\begin{array}{l}\text { - Per packet overhead is } \\
\text { more. } \\
\text { - Suffers from RTS } \\
\text { oscillations }\end{array}$ \\
\hline 7. & $\begin{array}{l}\text { Collision Hidden } \\
\text { Detection } \\
\text { Algorithm }\end{array}$ & $\begin{array}{l}\text { - In CARA-HD as the several } \\
\text { contending stations increases the } \\
\text { performance gap between them } \\
\text { becomes more significant }\end{array}$ & $\begin{array}{l}\text { - Performance degrades } \\
\text { in the presence of } \\
\text { hidden terminals. }\end{array}$ \\
\hline 8. & $\begin{array}{l}\text { Semi Open Loop } \\
\text { rate adaptation } \\
\text { algorithm }\end{array}$ & $\begin{array}{l}\text { - Quickly and accurately adapts rate } \\
\text { in MIMO system while minimizing } \\
\text { system overhead. }\end{array}$ & $\begin{array}{l}\text { - } \begin{array}{l}\text { More prone to errors } \\
\text { due to inaccuracies in } \\
\text { system model }\end{array} \\
\end{array}$ \\
\hline 9. & $\begin{array}{lr}\text { COMP } & \text { Rate } \\
\text { Adaption algorithm }\end{array}$ & $\begin{array}{l}\text { Better supports multiplexing } \\
\text { transmission and expands the } \\
\text { coverage of high data rate services }\end{array}$ & $\begin{array}{l}\text { - Increased overheads } \\
\text { - Huge data need to be } \\
\text { transferred between } \\
\text { eNode-B's for it to } \\
\text { operate. }\end{array}$ \\
\hline 10. & $\begin{array}{l}\text { Carrier } \\
\text { Aggregation } \\
\text { Algorithm }\end{array}$ & $\begin{array}{l}\text { - Supports aggregation of } 5 \text { carriers } \\
\text { in both UL and DL. } \\
\text { - Increases overall transmission } \\
\text { bandwidth of the system. }\end{array}$ & $\begin{array}{l}\text { - Inter-carrier load } \\
\text { balancing issues } \\
\text { Aggregation only } 5 \\
\text { component carriers }\end{array}$ \\
\hline
\end{tabular}




\section{Open Issues and Future Scope:}

It has been observed from the literature survey that many proposals have been published on LTE-A. But still there is a need of research to be carried in LTE-A as this technology is not fully matured so open issues and future scope are also raised in this section. In order to allow dividing the load more quickly between TDD and FDD frequencies this work provides the future possibility to aggregate TDD and FDD carriers jointly. This will allow the network to boost the UE throughput independently from UE in the cell. Also as assigning more spectrums to the user increases the data rate so in future spectrum could be increased or aggregated carriers may also be increased by using spectrum less than $20 \mathrm{MHz}$ per carrier component. Also Inter-carrier load balancing issues in carrier aggregation needs to be resolved and hence provides researchers an open field to work upon.

\section{Conclusion}

In this paper a survey of rate adaptation algorithms for effective resource utilization in LTE advance has been presented. Rate Adaptation Algorithms determines the optimal data transmission rate appropriate for current wireless channel conditions. RAA's in LTE advance enables selection of correct rate to maximize the performance of wireless device. Evaluation studies have shown that CoMP RAA may greatly enhance the cell-edge user experience. Carrier aggregation is an attractive RAA as it allows operators to deploy a system with extended bandwidth by aggregating several smaller component carriers while providing backward compatibility to legacy users. From this survey it may be concluded that for effective and efficient utilization of resources like bandwidth and power there is still a need of more research to be carried out. This paper will act as a ready reference for the researchers involved in the development of future releases of LTE based systems.

\section{References}

[1] T. Martin, "802.11b/g/n Wireless Networks Classification of rate adaptation algorithms", IEEE, (2012).

[2] A. Z. Yonis, M. F. L. Abdullah and M. F. Ghanim, "Effective Carrier Aggregation on the LTE-Advanced Systems", International Journal of Advanced Science and Technology, vol.41, (2012).

[3] D. Lee, H. Se and, E. Hardoui, "Coordinated Multipoint Transmission and Reception in LTE-Advanced: Deployment Scenarios and Operational Challenges", (2012).

[4] H Lee S. Wahid and K. Mossier, "A survey of RRM for spectrum aggregation in LTE advanced", IEEE Communications Survey tutorials, (2014).

[5] A. Annamalai1, R. C. Palat and J. Matyjas, "Estimating Ergodic Capacity of Cooperative Analog Relaying under Different Adaptive Source Transmission Techniques", (2011).

[6] C. Wang F. Haider and Yang D. Yuan, "Cellular architecture and key technologies for 5G wireless communication networks", (2014).

[7] K. I. Pedersen, F. F. C. Rosa, H. N. Luis, G. U. Garda and Y. Wang, “Carrier Aggregation for LTE advanced functionality and performance aspects", IEEE Communications Magazine, (2011).

[8] W. Santipach and M.L Honig, "Achievable rates for MIMO fading channels with limited feedback and linear receivers", IEEE eight international symposium and spread spectrum techniques and applications.

[9] P.A.K. Acharya, A. Sharma, E.M. Belding, K.C. Almeroth and K. Papagiannaki, "Rate adaptation in congested wireless networks through real-time measurements", Mobile Computing, IEEE, (2010).

[10] S. H.Y. Wong, H. Yang, S. Lu and V. Bharghavan, "Robust Rate Adaptation in Wireless Networks", Proc. of ACMMOBICOM, (2006).

[11] M.F L. Abdullah and A.Z. Yonis, "Design and Implementation of New Non-Contiguous Carrier Aggregation in Release 10", 1st IEEE International Symposium on Telecommunication Technologies, (2012). 

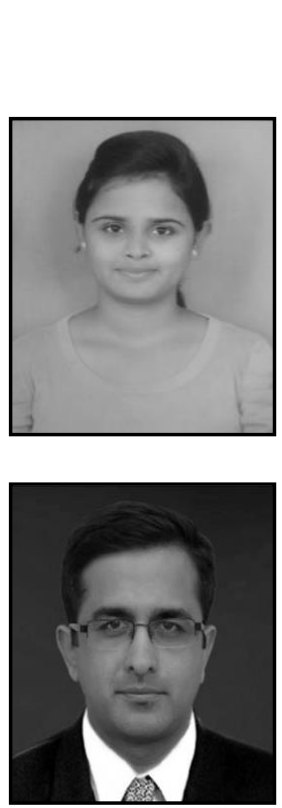

\section{Authors}

Diksha Duggal was born on $23^{\text {rd }}$ June, 1992 in Jalandhar, Punjab, INDIA. She completed B.Tech. in Electronics and Communication with Distinction from D.A.V Institute of Engineering and Technology, Jalandhar. She is currently persuing M.Tech. in Communication from Guru Nanak Dev University, RC Jalandhar. Her research interests are in the area of Wireless Communication \& Networks and Optical communication with emphasis on resource utilization in LTE Advance and performance analysis of carrier aggregation technique in LTE-A.

Jyoteesh Malhotra was born in Jalandhar, Punjab, INDIA. He completed B.Engg. with Distinction from P.R.M Institute of Technology \& Research, Amravati and M.Tech. with University Gold Medal from Guru Nanak Dev Engineering College, Ludhiana. He received PhD from Panjab Technical University in recognition to his contribution in the field of Wireless Communication \& Networks. He joined Guru Nanak Dev University Regional Campus at Jalandhar in July 2007 where he is currently Associate Professor and Head of ECE \& CSE Departments. His research interests are in the broad area of Pervasive Communication systems and Networks with emphasis on Statistical modeling of Fading Channels, Fading mitigation techniques, Optimization of High data rate Optical and wireless Communication Systems and Enhancement of QoS aware Wireless networks and Wireless Security. Dr. Malhotra has published and presented more than 100 technical papers in scientific journals and international conferences and authored 02 books. He is a life member of Indian Society for Technical Education (I.S.T.E.) and Editorial Board of many International Journals of repute. 
International Journal of Future Generation Communication and Networking Vol. 8, No. 3 (2015) 